\title{
Asymptotic normality of recursive estimators under strong mixing conditions
}

\author{
Amiri Aboubacar * \\ September 14, 2018
}

\begin{abstract}
The main purpose of this paper is to estimate the regression function by using a recursive nonparametric kernel approach. We derive the asymptotic normality for a general class of recursive kernel estimate of the regression function, under strong mixing conditions. Our purpose is to extend the work of Roussas and Tran [17] concerning the Devroye-Wagner estimate.
\end{abstract}

Keywords. Recursive kernel estimators, regression function, strong mixing processes, asymptotic normality.

\section{Introduction}

In this paper we consider nonparametric sequential estimation of a regression functional, for dependent observations. Regression function estimation is an important problem in data analysis and remains a subject of hight interest, which covers many applied fields such as prediction, econometrics, decision theory, classification, communications and control systems. The literature on this topic is still growing and some relevant work on this subject include the monographs by Prakasa Rao[14], Györfi et al. [10] and Yoshihara [21], while more

\footnotetext{
${ }^{*}$ Université Lille Nord de France, Université Lille 3, Laboratoire EQUIPPE EA 4018, Villeneuve d'Ascq, France. aboubacar.amiri@univ-lille3.fr.
} 
recent results are presented in, for example, the books by Györfi et al. [11 and Bosq and Blanke [4]. Sequential estimation is achieved with the use of recursive estimators, typically kernel ones, and the purpose of this paper is to study a certain class of them. An estimator is said to be 'recursive' if its value calculated from the first $n$ observations, say $f_{n}$, is only a function of $f_{n-1}$ and the $n^{\text {th }}$ observation. In this way, the estimator can be updated with each new observation added to the database. This recursive property is clearly useful in sequential investigations and also for a fairly large sample size, since addition of a new observation means the non-recursive estimators must be entirely recomputed. Besides, we are required to store extensive data in order to re-calculate them.

The first kernel recursive regression estimator was introduced by Ahmad and Lin [1] taking the form

$$
r_{n}^{A L}(x):=\frac{\sum_{i=1}^{n} Y_{i} K\left(\frac{x-X_{i}}{h_{i}}\right)}{\sum_{i=1}^{n} K\left(\frac{x-X_{i}}{h_{i}}\right)},
$$

which is a recursive version of the Nadaraya-Watson estimate. Also Devroye and Wagner [7] propose the recursive estimator of the form

$$
r_{n}^{D W}(x):=\frac{\sum_{i=1}^{n} \frac{Y_{i}}{h_{i}} K\left(\frac{x-X_{i}}{h_{i}}\right)}{\sum_{i=1}^{n} \frac{1}{h_{i}} K\left(\frac{x-X_{i}}{h_{i}}\right)} .
$$

In the literature $r_{n}^{A L}(x)$ and $r_{n}^{D W}(x)$ are respectively the so-called recursive and semi-recursive estimators. Various results on the latter estimators were established in an independent and identically distributed (i.i.d.) case, by many authors, we cite, among many others, Ahmad and Lin [1, Devroye [6], Greblicki and Pawlak [9], Krzyzak [12] and Walk [19]. In the dependent case, the majority of works are focused to Devroye-Wagner estimate. In a context of strong mixing processes, Roussas [16] gave the uniform almost sure convergence for $r_{n}^{D W}(x)$, and Roussas and Tran [17] showed its asymptotic normality. Under $\varphi$-mixing conditions, Qin [15] showed the asymptotic normality of $r_{n}^{D W}(x)$, and Wang and Liang [20] studied the almost uniform convergence for truncated versions of $r_{n}^{D W}(x)$ and $r_{n}^{A L}(x)$ in the same context. It should be noted that, unlike to the iid case, more results are only obtained for $r_{n}^{D W}(x)$ in dependent case. In particular no asymptotic normality has so far been established for $r_{n}^{A L}(x)$ in this context. Also we remark that, the approach utilized 
by Roussas and Tran [17] to establishing the asymptotic normality of $r_{n}^{D W}(x)$ cannot be generalized step by step to $r_{n}^{A L}(x)$. Indeed, the adaptation of their proof to $r_{n}^{A L}(x)$, needs to suppose that the sequence $\frac{1}{n} \sum_{i=1}^{n}\left(h_{i} / h_{n}\right)^{2 d}$ converges to a finite limit, for the study of a few covariance terms. The earlier condition is not satisfied by the popular choice $h_{n}=c n^{-\frac{1}{d+4}}$ for $d>3$. Also their proof uses the fact that for all $i=1, \ldots, n h_{n}<h_{i}$, while the same approach applied to $r_{n}^{A L}(x)$, leads to assume that $h_{n}>h_{i}$, which contradicts the optimal choice of $h_{n}$.

This paper deals with an extension of the work of Roussas and Tran [17] to the general family of recursive estimators introduced by Amiri [3], whose $r_{n}^{D W}(x)$ and $r_{n}^{A L}(x)$ are special cases. The paper is organized as follows. In the next section, we present our main assumptions and the result for regression estimation. The proof of the main result is postponed until Section 3 .

\section{Sequential regression estimation}

\section{$2.1 \quad$ Notation and assumptions}

Let $\left\{\left(X_{t}, Y_{t}\right), t \in \mathbb{N}\right\}$ be a sequence of random variables on probability space $(\Omega, \mathcal{F}, P)$, taking values in $\mathbb{R}^{d} \times \mathbb{R}^{d^{\prime}}\left(d \geq 1, d^{\prime} \geq 1\right)$, and having probability density function $f_{(X, Y)}$ with respect to the Lebesgue mesure. Assume that $m$ is a Borelian function on $\mathbb{R}^{d^{\prime}}$ into $\mathbb{R}$ such that $\omega \mapsto m^{2}\left(Y_{t}(\omega)\right)$ is $P$-integrable, and define the regression function as

$$
r(x):=\left\{\begin{array}{l}
\mathrm{E}\left(m\left(Y_{0}\right) \mid X_{0}=x\right)=\frac{\int_{\mathbb{R}^{d^{\prime}}} m(y) f_{(X, Y)}(x, y) d y}{f(x)}:=\frac{\varphi(x)}{f(x)}, \text { if } f(x)>0 \\
\operatorname{E} m\left(Y_{0}\right), \text { if } f(x)=0,
\end{array}\right.
$$

where $f$ is the probability density function of $X_{0}$. Note that the transformation $m$ is chosen by the statistician leading to multiple choices of estimation. Typical examples of $m$ are identity and polynomial functions to estimate respectively the usual regression and the conditional moments.

Throughout the paper we suppose that $f, \varphi \in C_{d}^{2}(b)$, where $C_{d}^{2}(b)$ denotes the set of twice-differentiable functions, with bounded second derivative. This condition is classical 
in the area of nonparametric estimation and has been used, among others, by Roussas and Tran [17], Bosq and Blanke [4].

To estimate the functional $r(x)$, we consider the general family of kernel regression estimators introduced in Amiri [3], defined by

$$
r_{n}^{\ell}(x):=\frac{\sum_{i=1}^{n} \frac{m\left(Y_{i}\right)}{h_{i}^{d \ell}} K\left(\frac{x-X_{i}}{h_{i}}\right)}{\sum_{i=1}^{n} \frac{1}{h_{i}^{d \ell}} K\left(\frac{x-X_{i}}{h_{i}}\right)}
$$

which can be computed recursively by

$$
r_{n}^{\ell}(x)=\frac{\left(\sum_{i=1}^{n-1} h_{i}^{d(1-\ell)}\right) \varphi_{n-1}^{\ell}(x)+\left(\sum_{i=1}^{n} h_{i}^{d(1-\ell)}\right) m\left(Y_{n}\right) K_{n}^{\ell}\left(x-X_{n}\right)}{\left(\sum_{i=1}^{n-1} h_{i}^{d(1-\ell)}\right) f_{n-1}^{\ell}(x)+\left(\sum_{i=1}^{n} h_{i}^{d(1-\ell)}\right) K_{n}^{\ell}\left(x-X_{n}\right)}
$$

where

$$
\varphi_{n}^{\ell}(x):=\frac{1}{\sum_{i=1}^{n} h_{i}^{d(1-\ell)}} \sum_{i=1}^{n} \frac{m\left(Y_{i}\right)}{h_{i}^{d \ell}} K\left(\frac{x-X_{i}}{h_{i}}\right), f_{n}^{\ell}(x):=\frac{1}{\sum_{i=1}^{n} h_{i}^{d(1-\ell)}} \sum_{i=1}^{n} \frac{1}{h_{i}^{d \ell}} K\left(\frac{x-X_{i}}{h_{i}}\right),
$$

and $K_{i}^{\ell}(\cdot):=\frac{1}{h_{i}^{d \ell} \sum_{j=1}^{i} h_{j}^{d(1-\ell)}} K\left(\frac{\dot{h_{i}}}{}\right)$. Our class of estimates includes the popular kernel recursive estimators $r_{n}^{A L}(x)$ and $r_{n}^{D W}(x)$, corresponding to the cases $\ell=0$ and $\ell=1$, respectively.

At this point, we make some assumptions and give the main theorem. Throughout this paper the kernel $K$ is assumed to satisfy the following conditions.

\section{Assumption H1.}

(i) $K: \mathbb{R}^{d} \mapsto \mathbb{R}$ is bounded, symmetric and positive function such that $\int_{\mathbb{R}^{d}} K(t) d t=1$;

(ii) $\lim _{\|x\| \rightarrow+\infty}\|x\|^{d} K(x)=0$;

(iii) $\int_{\mathbb{R}^{d}}\left|v_{i} v_{j}\right| K(v) d v<\infty, \quad i, j=1, \ldots, d$. 
Assume the sequence $h_{n}$ satisfies the following conditions.

\section{Assumption H2.}

(i) $h_{n} \downarrow 0, n h_{n}^{d+2} \rightarrow \infty$;

(ii) For all $r \in]-\infty, d+2], B_{n, r}:=\frac{1}{n} \sum_{i=1}^{n}\left(\frac{h_{i}}{h_{n}}\right)^{r} \rightarrow \beta_{r}>0$ as $n \rightarrow \infty$;

(iii) For each sequence of integers $u_{n}$ and $v_{n}$ such that $u_{n} \sim v_{n}$, then $h_{u_{n}} \sim h_{v_{n}}$. 1

\section{Assumption H3.}

(i) The process $\left(X_{t}\right)$ is $\alpha$-mixing with

$$
\alpha_{X}(k) \leq \gamma k^{-\rho}, \quad k \geq 1, \gamma>0 \text { and } \rho>\max \left(2, \frac{d+2}{2}\right) ;
$$

(ii) For each couple $(s, t), \quad s \neq t$, the random vector $\left(X_{s}, X_{t}\right)$ admits a probability density function $f_{\left(X_{s}, X_{t}\right)}$ such that $\sup _{|s-t| \geq 1}\left\|g_{s, t}\right\|_{\infty}<\infty$, where $g_{s, t}(\cdot, \cdot):=f_{\left(X_{s}, X_{t}\right)}(\cdot, \cdot)-$ $f(\cdot) f(\cdot)$.

\section{Assumption H4.}

(i) The function $\mathrm{E}\left(m^{2}(Y) \mid X_{0}=\cdot\right) f(\cdot)$ is both continuous and bounded away from zero at $x$;

(ii) There exist $\lambda>0, \theta>0$ such that $\operatorname{E} \exp \left(\lambda\left|m\left(Y_{0}\right)\right|^{\theta}\right)<\infty$;

(iii) For each $k \neq k^{\prime}$, the random vector $\left(X_{k}, Y_{k}, X_{k^{\prime}}, Y_{k^{\prime}}\right)$ admits a probability density function $f_{\left(X_{k}, Y_{k}, X_{k^{\prime}}, Y_{k^{\prime}}\right)}$, such that $\sup _{\left|k-k^{\prime}\right| \geq 1} \sup _{(s, t) \in \mathbb{R}^{2 d}} \int_{\mathbb{R}^{d^{\prime}}} \int_{\mathbb{R}^{d^{\prime}}}\left|G_{k, k^{\prime}}(s, u, t, v)\right| d u d v<\infty$, where

$$
G_{k, k^{\prime}}(\cdot, \cdot, \cdot, \cdot)=f_{\left(X_{k}, Y_{k}, X_{k^{\prime}}, Y_{k^{\prime}}\right)}(\cdot, \cdot, \cdot, \cdot)-f_{(X, Y)}(\cdot, \cdot) f_{(X, Y)}(\cdot, \cdot) .
$$

\footnotetext{
${ }^{1}$ If $a_{n}$ and $b_{n}$ are two real sequences, $a_{n} \sim b_{n}$ means that the ratio $a_{n} / b_{n}$ converges 1
} 
Assumptions H.1 and $\mathbf{H . 3}$ are classical in a nonparametric estimation field and they are the same as those classically used in the nonrecursive case. The first is satisfied by Gaussian and Eipanechnikov kernels, while the latter is checked by linear processes, as soon as $f$ is bounded. Note that H.1(i)-(ii) are technical conditions, the first allows the cancellation of the first-order term of Taylor development in the computation of the bias term, while the latter ensures the existence of the second-order term. Much more should be said about assumption H.2. This latter is particular to the recursive problem and is clearly unrestrictive since the choice $h_{n}=C_{n} n^{-\nu}$, with $C_{n} \downarrow c>0$, and $0<\nu<1$ is a typical example of bandwidth satisfying H.2. Concerning H.4, the condition H.4(ii) is clearly checked if $m$ is a bounded function, and implies that

$$
\mathrm{E}\left(\max _{1 \leq i \leq n}\left|m\left(Y_{i}\right)\right|^{p}\right)=O\left((\ln n)^{p / \theta}\right), \text { for all } p \geq 1, n \geq 2 .
$$

The earlier condition was used by Bosq and Cheze-Payaud [5] to study the mean square error of the Nadaraya-Watson estimator. Assumption H.4(iii) was used by Roussas and Tran [17] to study the asymptotic normality of $r_{n}^{D W}(x)$. Now, we can give the main result.

\subsection{Main result}

Let us set

$$
B_{n}=h_{n}^{2} \frac{\beta_{d(1-\ell)+2}}{\beta_{d(1-\ell)}} \frac{1}{2} \sum_{1 \leq i, j \leq d}\left(\frac{\partial^{2} r(x)}{\partial x_{i} \partial x_{j}}+2 \frac{\partial \ln f(x)}{\partial x_{i}} \frac{\partial r(x)}{\partial x_{j}}\right) \int_{\mathbb{R}^{d}} v_{i} v_{j} K(v) d v .
$$

The pointwise asymptotic gaussian distribution for our class of nonparametric recursive regression estimate is given in Theorem 1 below, and will be proved in Section 3 .

Theorem 1 When the assumptions $\boldsymbol{H . 1}-\boldsymbol{H . 4}$, hold, if for all $p>0,(\ln n)^{\frac{1}{\theta}} h_{n}^{p} \rightarrow 0$, as $n \rightarrow \infty$, then

$$
\sqrt{n h_{n}^{d}}\left[r_{n}^{\ell}(x)-r(x)-B_{n}\right] \stackrel{\mathcal{L}}{\rightarrow} \mathcal{N}\left[0, \frac{\sigma_{\ell}^{2}(x) V(x)}{f^{2}(x)}\right] \text {, as } n \rightarrow \infty,
$$

for all $x$ such that $f(x)>0$, where

$$
\sigma_{\ell}^{2}(x)=\frac{\beta_{d(1-2 \ell)}}{\beta_{d(1-\ell)}^{2}} f(x) \int_{\mathbb{R}^{d}} K^{2}(x) d x \text { and } V(x)=E\left[m^{2}\left(Y_{0}\right) \mid X_{0}=x\right]-r^{2}(x) .
$$


One may derive a simpler version of Theorem 1 by using an additional assumption which allows the cancellation of the bias term $B_{n}$.

Corollary $\mathbf{1}$ Under the assumptions $\boldsymbol{H . 1}-\boldsymbol{H . 4}$ and if $n h_{n}^{d+4} \rightarrow 0$ as $n \rightarrow 0$, then

$$
\sqrt{n h_{n}^{d}}\left[r_{n}^{\ell}(x)-r(x)\right] \stackrel{\mathcal{L}}{\rightarrow} \mathcal{N}\left[0, \frac{\sigma_{\ell}^{2}(x) V(x)}{f^{2}(x)}\right], \text { as } n \rightarrow \infty
$$

for all $x$ such that $f(x)>0$.

Corollary 1 is an extension of the Rousssas-Tran's [17] result on Devroye-Wagner estimate to the general family of recursive estimators $r_{n}^{\ell}(x)$ for which the Devroye-Wagner estimate is especial case. The condition $n h_{n}^{d+4} \rightarrow 0$ as $n \rightarrow 0$, implies that $(\ln n)^{\frac{1}{\theta}} h_{n}^{p} \rightarrow 0$, for all $p>0$, and it is satisfied by the choice $h_{n}=C_{n} n^{-\nu}$, with $C_{n} \downarrow c>0$ and $1 /(d+4)<\nu<1 /(d+2)$. Let us mention that $\mathbf{H . 2 ( i i i ) ~ w i l l ~ p l a y ~ a ~ k e y ~ r o l e ~ i n ~ o u r ~ m e t h o d o l o g y , ~ i n ~ p a r t i c u l a r ~ w h e n ~ w e ~}$ prove the negligibility of some covariance terms for $0 \leq \ell \leq(d-2)^{+} / 2$, but it is not necessary if $\ell>1 / 2$. Also if $\ell>1 / 2$, our results can be established for $\rho>2$. So, we observe that the estimators built with 'small' values of $\ell$ allow some restrictions on the smooth parameter $h_{n}$ and the strong mixing coefficient. However, as shown in Amiri [2], these estimators are preferable than those built with 'large' $\ell$ in terms of small variance criterion.

In practice, the constants of variance appearing in Theorem 1 need to be estimated. To this end, one may consider the simple Gaussian kernel and replace $f(x)$ by $f_{n}^{\ell}(x)$. There are many possibilities for constructing a consistent conditional variance estimate, one may use the functional kernel regression technique.

In order to prove Theorem 1, let us consider using the following decomposition.

$$
r_{n}^{\ell}(x)-r(x)=\left[\tilde{r}_{n}^{\ell}(x)-r(x)\right]+\left[r_{n}^{\ell}(x)-\tilde{r}_{n}^{\ell}(x)\right]
$$

where $\tilde{r}_{n}^{\ell}(x)=\tilde{\varphi}_{n}^{\ell}(x) / f_{n}^{\ell}(x), \tilde{\varphi}_{n}^{\ell}(x)$ being a truncated version of $\varphi_{n}^{\ell}(x)$ defined by

$$
\tilde{\varphi}_{n}^{\ell}(x)=\frac{1}{\sum_{i=1}^{n} h_{i}^{d(1-\ell)}} \sum_{i=1}^{n} \frac{Y_{i}}{h_{i}^{d \ell}} \mathbf{1}_{\left\{\left|Y_{i}\right| \leq b_{n}\right\}} K\left(\frac{x-X_{i}}{h_{i}}\right),
$$

with $b_{n}$, a sequence of real numbers which goes to $+\infty$ as $n \rightarrow \infty$. Next, we need the following preliminary lemmas.

Lemma 1 When the assumptions $\boldsymbol{H . 1}$ and $\boldsymbol{H} .2$ hold, then for all $\ell \in[0,1]$ 
(a)

$$
h_{n}^{-4}\left[E f_{n}^{\ell}(x)-f(x)\right]^{2} \longrightarrow\left[\frac{\beta_{d(1-\ell)+2}}{\beta_{d(1-\ell)}}\right]^{2} b_{f}^{2}(x) \text { as } n \rightarrow \infty
$$

(b)

$$
h_{n}^{-4}\left[E \varphi_{n}^{\ell}(x)-\varphi(x)\right]^{2} \longrightarrow\left[\frac{\beta_{d(1-\ell)+2}}{\beta_{d(1-\ell)}}\right]^{2} b_{\varphi}^{2}(x) \text { as } n \rightarrow \infty,
$$

where, if $h \in C_{d}^{2}(b)$, we set

$$
b_{h}(x):=\frac{1}{2} \sum_{1 \leq i, j \leq d} \frac{\partial^{2} h}{\partial x_{i} \partial x_{j}}(x) \int_{\mathbb{R}^{d}} v_{i} v_{j} K(v) d v .
$$

(c) Moreover if $\mathbf{H . 3}$ holds, then

$$
n h_{n}^{d} \operatorname{Varf}_{n}^{\ell}(x) \longrightarrow \sigma_{\ell}^{2}(x), \text { as } n \rightarrow \infty,
$$

for all $x$ such that $f(x)>0$.

Proof. The results (a) and (c) of Lemma 1 are obtained in Amiri [2], while (b) can be established in the same manner as (a) by substituting $f$ by $\varphi$.

Lemma $\mathbf{2}$ When the assumptions $\boldsymbol{H . 1}-\boldsymbol{H} .4$ hold, then for all $\ell \in[0,1]$

(a)

$$
n h_{n}^{d} \operatorname{Var}_{\tilde{\varphi}_{n}^{\ell}}(x) \longrightarrow \sigma_{\ell}^{2}(x)\left[r^{2}(x)+V(x)\right] \text {, as } n \rightarrow \infty \text {. }
$$

(b)

$$
n h_{n}^{d} \operatorname{Cov}\left[f_{n}^{\ell}(x), \tilde{\varphi}_{n}^{\ell}(x)\right] \rightarrow \sigma_{\ell}^{2}(x) r(x) \text { as } n \rightarrow \infty .
$$

Proof.

(a) Let us set

$V_{n}^{*}=\sum_{k=1}^{n} \mathrm{E} Z_{k, n}^{* 2}$ where $Z_{i, n}^{*}=W_{n, i}-\mathrm{E} W_{n, i}$, with $W_{n, i}:=\frac{K\left(\frac{x-X_{i}}{h_{i}}\right) m\left(Y_{i}\right) \mathbf{1}_{\left\{\left|m\left(Y_{i}\right)\right| \leq b_{n}\right\}}}{h_{i}^{d \ell}}$.

The variance of $\tilde{\varphi}_{n}^{\ell}(x)$ can be decomposed in variance and covariance terms as

$$
\operatorname{Var} \tilde{\varphi}_{n}^{\ell}(x)=\frac{1}{n^{2} h_{n}^{2 d(1-\ell)} B_{n, d(1-\ell)}^{2}}\left[V_{n}^{*}+\sum_{k=1}^{n} \sum_{k \neq k^{\prime}}^{n} \operatorname{Cov}\left(Z_{k, n}, Z_{k^{\prime}, n}\right)\right] .
$$


Concerning the variance term one may write

$$
\begin{aligned}
\frac{V_{n}^{*}}{n h_{n}^{d(1-2 \ell)} B_{n, d(1-2 \ell)}} & =\frac{n h_{n}^{d}}{\left(\sum_{i=1}^{n} h_{i}^{d(1-\ell)}\right)^{2}} \sum_{k=1}^{n}\left\{h_{k}^{-2 d \ell} \mathrm{E} K^{2}\left(\frac{x-X_{0}}{h_{k}}\right) m^{2}\left(Y_{0}\right)\right. \\
& -h_{k}^{-2 d \ell} \mathrm{E}^{2}\left(\frac{x-X_{0}}{h_{k}}\right) m^{2}\left(Y_{0}\right) \mathbf{1}_{\left\{\left|m\left(Y_{0}\right)\right|>b_{n}\right\}} \\
& \left.-\mathrm{E}^{2} K\left(\frac{x-X_{0}}{h_{k}}\right) m\left(Y_{0}\right) \mathbf{1}_{\left\{\left|m\left(Y_{i}\right)\right| \leq b_{n}\right\}}\right\}=: D_{1}+D_{2}+D_{3} .
\end{aligned}
$$

Assumptions H.4 $($ ii $)$, (iii), the dominated convergence theorem and Bochner's lemma imply that

$$
\int_{\mathbb{R}^{d}} \frac{1}{h_{k}^{d}} K^{2}\left(\frac{x-u}{h_{k}}\right)\left[V(u)+r^{2}(u)\right] f(u) d u \rightarrow f(x)\left[V(x)+r^{2}(x)\right]\|K\|_{2}^{2} \text {, as } k \rightarrow \infty .
$$

On account of the above, assumption H.2(ii) and the Toeplitz lemma allow to deduce that

$D_{1}=\frac{n h_{n}^{d} \sum_{k=1}^{n}\left[h_{k}^{d(1-2 \ell)} \int_{\mathbb{R}^{d}} \frac{1}{h_{k}^{d}} K^{2}\left(\frac{x-u}{h_{k}}\right)\left[V(u)+r^{2}(u)\right] f(u) d u\right]}{\left(\sum_{i=1}^{n} h_{i}^{d(1-\ell)}\right)^{2}} \rightarrow \sigma_{\ell}^{2}(x)\left[V(x)+r^{2}(x)\right]$,

as $n \rightarrow \infty$. Concerning the term $D_{2}$, if $b_{n}=(\delta \ln n)^{\frac{1}{\theta}}$ with $\delta>\frac{2}{\lambda}$, then using the assumptions H.2(ii) and H.4(ii), with the help of Markov's inequality, we have

$$
\begin{aligned}
\left|D_{2}\right| & \leq \frac{\|K\|_{\infty}^{2}\left\{\mathrm{Em}^{4}\left(Y_{0}\right) P\left(\left|m\left(Y_{0}\right)\right|>b_{n}\right)\right\}^{\frac{1}{2}} n h_{n}^{d} \sum_{k=1}^{n} h_{k}^{-2 d \ell}}{\left(\sum_{i=1}^{n} h_{i}^{d(1-\ell)}\right)^{2}} \leq \frac{\|K\|_{\infty}^{2}\left\{\mathrm{Em}^{4}\left(Y_{0}\right) P\left(\left|m\left(Y_{0}\right)\right|>b_{n}\right)\right\}^{\frac{1}{2}} B_{n,-2 d \ell}}{h_{n}^{d} B_{n, d(1-\ell)}^{2}} \\
& =O\left[\frac{\exp \left(-\frac{\lambda b_{n}^{\theta}}{2}\right)(\ln n)^{\frac{2}{\theta}} B_{n,-2 d \ell}}{h_{n}^{d} B_{n, d(1-\ell)}^{2}}\right] \rightarrow 0, \text { as } n \rightarrow \infty .
\end{aligned}
$$

Next for the last term $D_{3}$, from H.2(i)-(iii) and the logarithmic choice of $b_{n}$, one may write

$$
\left|D_{3}\right| \leq \frac{b_{n}^{2} n h_{n}^{d}}{n^{2} h_{n}^{2 d(1-\ell)} B_{n, d(1-\ell)}^{2}} \sum_{k=1}^{n} h_{k}^{-2 d \ell}\left(\mathrm{E} K\left(\frac{x-X_{i}}{h_{i}}\right)\right)^{2}=O\left(h_{n} b_{n}^{2}\right) \rightarrow 0, \text { as } n \rightarrow+\infty .
$$

Therefore

$$
V_{n}^{*} \sim n h_{n}^{d(1-2 \ell)} \beta_{d(1-2 \ell)} f(x)\left[V(x)+r^{2}(x)\right] \int_{\mathbb{R}^{d}} K^{2}(u) d u, \text { as } n \rightarrow \infty .
$$


It follows that

$$
\frac{n h_{n}^{d} V_{n}^{*}}{n^{2} h_{n}^{2 d(1-\ell)} B_{n, d(1-\ell)}^{2}} \rightarrow \frac{\beta_{d(1-2 \ell)} f(x)\left[V(x)+r^{2}(x)\right]}{\beta_{d(1-\ell)}^{2}} \int_{\mathbb{R}^{d}} K^{2}(u) d u, \text { as } n \rightarrow \infty .
$$

Now, let us show that the covariance term of $\operatorname{Var} \tilde{\varphi}_{n}^{\ell}(x)$ is negligible. To this end, define a sequence $c_{n}$ of real numbers tending to zero as $n$ goes to infinity, and write

$$
\begin{aligned}
\frac{\sum_{k=1}^{n} \sum_{k \neq k^{\prime}} \sum_{k^{\prime}=1}^{n} \operatorname{Cov}\left(Z_{k, n}, Z_{k^{\prime}, n}\right)}{n^{2} h_{n}^{2 d(1-\ell)} B_{n, d(1-\ell)}^{2}} & \leq \frac{2\left(\sum_{i=1}^{n} \sum_{i>j}^{n}\left|A_{i, j}\right| 1_{\left\{1 \leq i-j \leq c_{n}\right\}}+\sum_{i=1}^{n} \sum_{i>j} \sum_{j=1}^{n}\left|A_{i, j}\right| 1_{\left\{c_{n}+1 \leq i-j \leq n-1\right\}}\right)}{\left(\sum_{i=1}^{n} h_{i}^{d(1-\ell)}\right)^{2}} \\
& \leq \frac{2\left(\sum_{i=1}^{c_{n}} \sum_{p=1}^{n} A_{i+p, p}+\sum_{i=c_{n+1}}^{n-1} \sum_{p=1}^{n} A_{i+p, p}\right)}{\left(\sum_{i=1}^{n} h_{i}^{d(1-\ell)}\right)^{2}}:=L_{1}+L_{2},
\end{aligned}
$$

where

$$
A_{i+p, p}=\frac{\left|\operatorname{Cov}\left[K\left(\frac{x-X_{i+p}}{h_{i+p}}\right) m\left(Y_{i+p}\right) \mathbf{1}_{\left\{\left|m\left(Y_{i+p}\right)\right| \leq b_{n}\right\}}, K\left(\frac{x-X_{p}}{h_{p}}\right) m\left(Y_{p}\right) \mathbf{1}_{\left\{\left|m\left(Y_{p}\right)\right| \leq b_{n}\right\}}\right]\right|}{h_{i+p}^{d \ell} h_{p}^{d \ell}} .
$$

On one hand, the Billingsley inequality (see e.g., Bosq Blanke [4]) implies that

$$
A_{i+p, p} \leq 4 b_{n}^{2} \alpha_{X}(k)\|K\|_{\infty}^{2} h_{i+p}^{-d \ell} h_{p}^{-d \ell}
$$

and then, it follows from assumptions $\mathbf{H 2 ( i i ) ~ a n d ~ H . 4 ( i v ) ~ t h a t ~}$

$$
\begin{aligned}
L_{2} & \leq \frac{8 b_{n}^{2} \sum_{k=c_{n}+1}^{n-1} \sum_{p=1}^{n} \alpha_{X}(k) h_{p+k}^{-d \ell} h_{p}^{-d \ell}}{\|K\|_{\infty}^{2}\left(\sum_{i=1}^{n} h_{i}^{d(1-\ell)}\right)^{2}} \leq \frac{8 b_{n}^{2} \gamma\|K\|_{\infty}^{2} \sum_{k=c_{n}}^{n-1} \sum_{p=1}^{n} k^{-\rho} h_{p+k}^{-d \ell} h_{p}^{-d \ell}}{\left(\sum_{i=1}^{n} h_{i}^{d(1-\ell)}\right)^{2}} \leq \frac{8 b_{n}^{2} \gamma\|K\|_{\infty}^{2} \frac{h_{n}^{-2 d \ell} c_{n}^{-\rho+1}}{\rho-1} \sum_{p=1}^{n}\left(\frac{h_{p}}{h_{n}}\right)^{-d \ell}}{\left(\sum_{i=1}^{n} h_{i}^{d(1-\ell)}\right)^{2}} \\
& \leq \frac{8 b_{n}^{2} \gamma\|K\|_{\infty}^{2} c_{n}^{1-\rho} B_{n,-d \ell}}{n h_{n}^{2 d} B_{n, d(1-\ell)}^{2}(\rho-1)} .
\end{aligned}
$$

Hence

$$
n h_{n}^{d} L_{2}=O\left(b_{n}^{2} c_{n}^{1-\rho} h_{n}^{-d}\right) .
$$

On the other hand, regarding about $L_{1}$, one has

$$
\begin{aligned}
A_{i+p, p} & =\left|\int_{\mathbb{R}^{d}} \int_{\mathbb{R}^{d}} \int_{\mathbb{R}^{d^{\prime}}} \int_{\mathbb{R}^{d^{\prime}}} K\left(\frac{x-s}{h_{i+p}}\right) K\left(\frac{x-t}{h_{p}}\right) \frac{m(u) \mathbf{1}_{\left\{|m(u)| \leq b_{n}\right\}} m(v) \mathbf{1}_{\left\{|m(v)| \leq b_{n}\right\}} G_{i+p, p}(s, u, t, v)}{\left(h_{i+p} h_{p}\right)^{d \ell}\left(\sum_{i=1}^{n} h_{i}^{d(1-\ell)}\right)^{2}} d s d t d u d v\right| \\
& \leq \frac{b_{n}^{2}\left(h_{k+p} h_{p}\right)^{d(1-\ell)} \sup _{\left|k-k^{\prime}\right| \geq 1(s, t) \in \mathbb{R}^{2 d}} \int_{\mathbb{R}^{d^{\prime}}} \int_{\mathbb{R}^{d^{\prime}}}\left|G_{k, k^{\prime}}(s, u, t, v)\right| d u d v}{\left(\sum_{i=1}^{n} h_{i}^{d(1-\ell)}\right)^{2}} .
\end{aligned}
$$


Then

$$
\begin{aligned}
L_{1} & \leq \frac{2 b_{n}^{2} \sum_{k=1}^{c_{n}} \sum_{p=1}^{n-k} h_{p+k}^{d(1-\ell)} h_{p}^{d(1-\ell)} \sup _{\left|k-k^{\prime}\right| \geq 1} \sup _{(s, t) \in \mathbb{R}^{2 d}} \int_{\mathbb{R}^{d^{\prime}}} \int_{\mathbb{R}^{d^{\prime}}}\left|G_{k, k^{\prime}}(s, u, t, v)\right| d u d v}{\left(\sum_{i=1}^{n} h_{i}^{d(1-\ell)}\right)^{2}} \\
& \leq \frac{2 b_{n}^{2} c_{n} \sum_{p=1}^{n} h_{p}^{2 d(1-\ell)} \sup _{\left|k-k^{\prime}\right| \geq 1} \sup _{(s, t) \in \mathbb{R}^{2 d}} \int_{\mathbb{R}^{d^{\prime}}} \int_{\mathbb{R}^{d^{\prime}}}\left|G_{k, k^{\prime}}(s, u, t, v)\right| d u d v}{\left(\sum_{i=1}^{n} h_{i}^{d(1-\ell)}\right)^{2}} .
\end{aligned}
$$

At this point, we distinguish two cases according to small and large values of $\ell$.

If $\ell \in\left[\left(\frac{d-2}{2 d}\right)^{+}, 1\right]$, then $2 d(1-\ell) \leq d+2$ replies $B_{n, 2 d(1-\ell)} \rightarrow \beta_{2 d(1-\ell)}<\infty$, as $n \rightarrow \infty$, because of $\mathbf{H . 2}(i i)$. It follows that

$$
L_{1} \leq \frac{2 b_{n}^{2} c_{n} B_{n, 2 d(1-\ell)} \sup _{\left|k-k^{\prime}\right| \geq 1} \sup _{(s, t) \in \mathbb{R}^{2 d}} \int_{\mathbb{R}^{d^{\prime}}} \int_{\mathbb{R}^{d^{\prime}}}\left|G_{k, k^{\prime}}(s, u, t, v)\right| d u d v}{n B_{n, d(1-\ell)}^{2}},
$$

which implies that

$$
n h_{n}^{d} L_{1}=O\left(b_{n}^{2} c_{n} h_{n}^{d}\right)
$$

Thus, when $c_{n}:=\left\lfloor h_{n}^{-\frac{2 d}{\rho}}\right\rfloor$, and $b_{n}=(\delta \ln n)^{\frac{1}{\theta}}$ with $\delta>\frac{2}{\lambda}$, then

$$
\frac{n h_{n}^{d}}{n^{2} h_{n}^{2 d(1-\ell)} B_{n, d(1-\ell)}^{2}} \sum_{k=1}^{n} \sum_{k \neq k^{\prime}}^{n} \operatorname{Cov}\left(Z_{k, n}, Z_{k^{\prime}, n}\right)=O\left(b_{n}^{2} h_{n}^{-\frac{d(2-\rho)}{\rho}}\right) \rightarrow 0, \text { as } n \rightarrow \infty
$$

since $\rho>2$. Now, if $d \geq 3, \quad \ell \in\left[0, \frac{d-2}{2 d}\left[\right.\right.$, then the term $L_{1}$ cannot be studied as previously, because assumption $\mathbf{H . 2 ( i i )}$ is not satisfied since $2 d(1-\ell)>d+2$. In this case, let us consider relation (2) and choose a real number $\xi$ such that $\frac{1}{\rho-1}<\xi \leq \frac{2}{d}$. Let us mention that $\xi$ exists only if $\rho>\frac{d+2}{2}$. Thus, we have the relation $d(\xi+1) \leq$ $d+2$, which implies that $B_{n, d(\xi+1)} \rightarrow \beta_{d(\xi+1)}<\infty$, as $n \rightarrow \infty$, by vertue of $\mathbf{H . 2}(i i)$. Next, since $h_{n}$ decreases on has $\sum_{i=1}^{n} h_{i}^{d(1-\ell)} \geq h_{1}^{-d \ell} \sum_{i=1}^{n} h_{i}^{d}$. It follows that

$$
\frac{c_{n} b_{n}^{2} \sum_{p=1}^{n} h_{p}^{2 d(1-\ell)}}{\left(\sum_{i=1}^{n} h_{i}^{d(1-\ell)}\right)^{2}} \leq \frac{c_{n} b_{n}^{2} h_{1}^{d(1-\xi-2 \ell)} n h_{n}^{d(\xi+1)} B_{n, d(\xi+1)}}{n^{2} h_{1}^{-2 d \ell} h_{n}^{2 d} B_{n, d}^{2}} \leq \frac{c_{n} b_{n}^{2} h_{1}^{d(1-\xi)} h_{n}^{d \xi} B_{n, d(\xi+1)}}{n h_{n}^{d} B_{n, d}^{2}}
$$

because $0 \leq \ell<\frac{d-2}{2 d} \Rightarrow 1-\xi-2 \ell>0$, as long as $\xi \leq \frac{2}{d}$. Therefore, from (2) we have

$$
n h_{n}^{d} L_{1}=O\left(c_{n} b_{n}^{2} h_{n}^{d \xi}\right)
$$


The choices $c_{n}:=\left\lfloor h_{n}^{-\frac{d(\xi+1)}{\rho}}\right\rfloor$ and $b_{n}=(\delta \ln n)^{\frac{1}{\theta}}$ with $\delta>\frac{2}{\lambda}$, imply the negligibility of the covariance term.

(b) Let us consider the decomposition

$$
\operatorname{Cov}\left[f_{n}^{\ell}(x), \tilde{\varphi}_{n}^{\ell}(x)\right]=\left[\sum_{i=1}^{n} h_{i}^{d(1-\ell)}\right]^{-2}\left[\sum_{i=1}^{n} A_{i i}+\sum_{i=1}^{n} \sum_{i \neq j}^{n} A_{i j}\right]:=F_{1}+F_{2} .
$$

where, for all integers $s, t$

$$
A_{s, t}:=\operatorname{Cov}\left[\frac{1}{h_{s}^{d \ell}} K\left(\frac{x-X_{s}}{h_{s}}\right), \frac{m\left(Y_{t}\right)}{h_{t}^{d \ell}} \mathbf{1}_{\left\{\left|m\left(Y_{i}\right) \leq b_{n}\right|\right\}} K\left(\frac{x-X_{t}}{h_{t}}\right)\right] .
$$

Next, we proceed as in the proof of (a) and find

$$
n h_{n}^{d} F_{1} \rightarrow \sigma_{\ell}^{2}(x) r(x), \text { and } n h_{n}^{d} F_{2} \rightarrow 0, \text { as } n \rightarrow \infty
$$

\section{Proof of main result}

Proof. To prove the main result, we show that the asymptotic distribution of the principal term $\left[\tilde{r}_{n}^{\ell}(x)-r(x)\right]$ is normal, while the residual term $\left[r_{n}^{\ell}(x)-\tilde{r}_{n}^{\ell}(x)\right]$ is negligible. First, observe that if $b_{n}=(\delta \ln n)^{\frac{1}{\theta}}$ with $\delta>\frac{2}{\lambda}$, then for all $\varepsilon>0$, we have

$$
P\left(\left|\varphi_{n}^{\ell}(x)-\tilde{\varphi}_{n}^{\ell}(x)\right|>\varepsilon / \sqrt{n h_{n}^{d}}\right) \leq P\left(\bigcup_{i=1}^{n}\left\{\left|Y_{i}\right|>b_{n}\right\}\right) \leq n P\left(\left|Y_{0}\right|>b_{n}\right) \leq \mathrm{E} e^{\lambda\left|m\left(Y_{0}\right)\right|^{\theta}} n^{1-\lambda \delta} .
$$

So, for all $\varepsilon>0, \sum_{n=1}^{\infty} P\left(\left|\varphi_{n}^{\ell}(x)-\tilde{\varphi}_{n}^{\ell}(x)\right|>\varepsilon / \sqrt{n h_{n}^{d}}\right)<\infty$, and the Borel-Cantelli lemma implies that

$$
\sqrt{n h_{n}^{d}}\left[r_{n}^{\ell}(x)-\tilde{r}_{n}^{\ell}(x)\right] \rightarrow 0 \text { a.s, as } n \rightarrow \infty .
$$

One may prove in the same manner that $f_{n}^{\ell}(x) \rightarrow f(x)$ a.s as $n \rightarrow \infty$. Next, we need to show that

$$
\sqrt{n h_{n}^{d}}\left[\tilde{r}_{n}^{\ell}(x)-B_{n}-r(x)\right] \stackrel{\mathcal{L}}{\rightarrow} \mathcal{N}\left[0, \frac{\beta_{d(1-2 \ell)}\|K\|_{2}^{2} V(x)}{\beta_{d(1-\ell)}^{2} f(x)}\right]
$$


as $n \rightarrow \infty$. To this end, we use the following representation

$$
\tilde{r}_{n}^{\ell}(x)-r(x)-B_{n}=\frac{1}{f_{n}^{\ell}(x) \mathrm{E} f_{n}^{\ell}(x)}\left[\begin{array}{c}
\mathrm{E} f_{n}^{\ell}(x) \\
-\mathrm{E} \tilde{\varphi}_{n}^{\ell}(x)
\end{array}\right]^{T}\left[\begin{array}{c}
\tilde{\varphi}_{n}^{\ell}(x)-\mathrm{E} \tilde{\varphi}_{n}^{\ell}(x) \\
f_{n}^{\ell}(x)-\mathrm{E} f_{n}^{\ell}(x)
\end{array}\right]+o\left(\frac{1}{\sqrt{n h_{n}^{d}}}\right) .
$$

Now, applying the Cramer-Wold device and remembering that $f_{n}^{\ell}(x) \stackrel{a . s}{\rightarrow} f(x)$, and $\mathrm{E} f_{n}^{\ell}(x) \rightarrow$ $f(x)$, as $n \rightarrow \infty$, the proof of the Theorem 1 is straightforward from the following claim:

$$
\sqrt{n h_{n}^{d}}\left[\begin{array}{c}
\tilde{\varphi}_{n}^{\ell}(x)-\mathrm{E} \tilde{\varphi}_{n}^{\ell}(x) \\
f_{n}^{\ell}(x)-\mathrm{E} f_{n}^{\ell}(x)
\end{array}\right] \stackrel{\mathcal{L}}{\rightarrow} \mathcal{N}_{2}\left\{0, \quad \sigma_{\ell}^{2}(x)\left[\begin{array}{lr}
V(x)+r^{2}(x) & r(x) \\
r(x) & 1
\end{array}\right]\right\} \text {, as } n \rightarrow \infty .
$$

This last convergence is equivalent to

$$
\sqrt{n h_{n}^{d}}\left\{\lambda_{1}\left[f_{n}^{\ell}(x)-\mathrm{E} f_{n}^{\ell}(x)\right]+\lambda_{2}\left[\tilde{\varphi}_{n}^{\ell}(x)-\mathrm{E} \tilde{\varphi}_{n}^{\ell}(x)\right]\right\} \stackrel{\mathcal{L}}{\rightarrow} \mathcal{N}\left[0, \Sigma_{\ell}^{2}(x)\right], \text { as } n \rightarrow \infty
$$

for each $\lambda_{1}, \lambda_{2} \in \mathbb{R}$ such that $\lambda_{1}+\lambda_{2} \neq 0$, where

$$
\Sigma_{\ell}^{2}(x):=\sigma_{\ell}^{2}(x)\left\{\lambda_{1}^{2}+2 \lambda_{1} \lambda_{2} r(x)+\lambda_{2}^{2}\left[V(x)+r^{2}(x)\right]\right\} .
$$

Hence, the main result will be completely proven if (3) were established. To this end, let us set

$$
\tilde{\Psi}_{n j}:=\lambda_{1} \Psi_{n j}+\lambda_{2} \Psi_{n j}^{\prime}
$$

where $\Psi_{n j}:=\left[\frac{h_{n}^{d(2 \ell-1)}}{n}\right]^{\frac{1}{2}} \frac{h_{j}^{-d \ell}}{B_{n, d(1-\ell)}}\left(V_{n j}-\mathrm{E} V_{n j}\right)$ and $\Psi_{n j}^{\prime}:=\left[\frac{h_{n}^{d(2 \ell-1)}}{n}\right]^{\frac{1}{2}} \frac{h_{j}^{-d \ell}}{B_{n, d(1-\ell)}}\left(W_{n j}-\mathrm{E} W_{n j}\right)$ with

$$
V_{n j}:=K\left(\frac{x-X_{j}}{h_{j}}\right) \text { and } W_{n j}:=K\left(\frac{x-X_{j}}{h_{j}}\right) m\left(Y_{j}\right) \mathbf{1}_{\left\{\left|m\left(Y_{j}\right)\right| \leq b_{n}\right\}} .
$$

Next, consider the sequences $\varsigma_{n}, \tau_{n}$, and $r_{n}$ defined as

$$
\tau_{n}:=\left\lfloor\tau_{0} \log n\right\rfloor, \quad \varsigma_{n}:=\left\lfloor\frac{\tau_{0} \sqrt{n h_{n}^{d}}}{(\log n)^{\varsigma_{0}}}\right\rfloor \text { and } r_{n}:=\left\lfloor\frac{n}{\varsigma_{n}+\tau_{n}}\right\rfloor \text {, with } \tau_{0}, \varsigma_{0}>0 .
$$

To establish (3), we use the classical Doob [8] methodology, which consists of splitting the term

$$
\sqrt{n h_{n}^{d}}\left\{\lambda_{1}\left[f_{n}^{\ell}(x)-f(x)\right]+\lambda_{2}\left[\tilde{\varphi}_{n}^{\ell}(x)-\varphi(x)\right]\right\}
$$


into large blocks separated by small blocks defined by

$$
\begin{aligned}
& T_{n m}=\sum_{j=k_{m}}^{k_{m}+\varsigma_{n}-1} \tilde{\Psi}_{n j} \text { (large blocks), } T_{n m}^{\prime}=\sum_{j=l_{m}}^{l_{m}+\tau_{n}-1} \tilde{\Psi}_{n j} \text { (small blocks), } \\
& T_{n r_{n}+1}^{\prime}=\sum_{j=\bar{N}+1}^{n} \tilde{\Psi}_{n j} \text { (rest of term) }
\end{aligned}
$$

where $\bar{N}:=r_{n}\left(\tau_{n}+\varsigma_{n}\right)$, and for $m=1, \ldots, r_{n}, k_{m}:=(m-1)\left(\varsigma_{n}+\tau_{n}\right)+1, \quad l_{m}:=(m-$ 1) $\left(\varsigma_{n}+\tau_{n}\right)+\varsigma_{n}+1$. Next, let us define the partial sums

$$
S_{n 1}=\sum_{m=1}^{r_{n}} T_{n m}, \quad S_{n 2}=\sum_{m=1}^{r_{n}} T_{n m}^{\prime} \text { and } S_{n 3}=T_{n r_{n}+1}^{\prime} .
$$

Thus, we can write

$$
\sqrt{n h_{n}^{d}}\left\{\lambda_{1}\left[f_{n}^{\ell}(x)-f(x)\right]+\lambda_{2}\left[\tilde{\varphi}_{n}^{\ell}(x)-\varphi(x)\right]\right\}=S_{n 1}+S_{n 2}+S_{n 3} .
$$

The goal is to prove that, $\mathrm{E} S_{n 2}^{2}$ and $\mathrm{E} S_{n 3}^{2}$ converge to zero, while the asymptotic distribution of $S_{n 1}$ is normal. First, observe that

$$
\begin{aligned}
\mathrm{E} S_{n 2}^{2} & =\sum_{m=1}^{r_{n}} \operatorname{Var}\left(T_{n m}^{\prime}\right)+2 \sum_{1 \leq i<j \leq r_{n}} \operatorname{Cov}\left(T_{n i}^{\prime}, T_{n j}^{\prime}\right) \\
& =\sum_{m=1}^{r_{n}} \sum_{i=l_{m}}^{l_{m}+\tau_{n}-1} \operatorname{Var} \tilde{\Psi}_{n i}+2 \sum_{m=1}^{r_{n}} \sum_{l_{m} \leq i<j \leq l_{m}+\tau_{n}-1} \operatorname{Cov}\left(\tilde{\Psi}_{n i}, \tilde{\Psi}_{n j}\right) \\
& +2 \sum_{1 \leq i<j \leq r_{n}} \sum_{s=l_{i}}^{l_{i}+\tau_{n}-1} \sum_{t=l_{j}}^{l_{j}+\tau_{n}-1} \operatorname{Cov}\left(\tilde{\Psi}_{n s}, \tilde{\Psi}_{n t}\right):=\Delta_{1}+\Delta_{2}+\Delta_{3} .
\end{aligned}
$$

The first term in (44), is decomposed as

$$
\Delta_{1}=\sum_{m=1}^{r_{n}} \sum_{i=l_{m}}^{l_{m}+\tau_{n}-1}\left[\lambda_{1}^{2} \operatorname{Var} \Psi_{n i}+\lambda_{2}^{2} \operatorname{Var} \Psi_{n i}^{\prime}+2 \lambda_{1} \lambda_{2} \operatorname{Cov}\left(\Psi_{n i}, \Psi_{n i}^{\prime}\right)\right]:=\Delta_{11}+\Delta_{12}+\Delta_{13}
$$

Since $h_{n}$ decreases, the choice of $b_{n}=(\delta \ln n)^{\frac{1}{\theta}}$ with $\delta>\frac{2}{\lambda}$, and $\theta>1 / \varsigma_{0}$ with the help of H2(iii) implies that

$$
\begin{aligned}
\Delta_{11}+\Delta_{12} & =\frac{h_{n}^{d(2 \ell-1)}}{n B_{n, d(1-\ell)}^{2}} \sum_{m=1}^{r_{n}} \sum_{j=l_{m}}^{l_{m}+\tau_{n}-1} h_{j}^{-2 d \ell}\left[\lambda_{1}^{2} \operatorname{Var} K\left(\frac{x-X_{j}}{h_{j}}\right)+\lambda_{2}^{2} \operatorname{Var} K\left(\frac{x-X_{j}}{h_{j}}\right) Y_{j} \mathbf{1}_{\left\{\left|m\left(Y_{j}\right)\right| \leq b_{n}\right\}}\right] \\
& \leq \frac{r_{n} \tau_{n}\left(1+b_{n}^{2}\right)\|K\|_{\infty}^{2} \max \left(\lambda_{1}^{2}, \lambda_{2}^{2}\right)}{n h_{n}^{d} B_{n, d(1-\ell)}^{2}} \rightarrow 0, \text { as } n \rightarrow \infty,
\end{aligned}
$$


and, similarly, we have $\Delta_{13} \leq \frac{2 \lambda_{1} \lambda_{2} b_{n} r_{n} \tau_{n}\|K\|_{\infty}^{2}}{n h_{n}^{d} B_{n, d(1-\ell)}^{2}} \rightarrow 0$, as $n \rightarrow \infty$. In the same manner and by also using the Cauchy-Schwartz's inequality, we get

$$
\Delta_{2} \leq \frac{r_{n} \tau_{n}^{2}\left(1+b_{n}\right)^{2}\|K\|_{\infty}^{2} \max \left(\lambda_{1}^{2}, \lambda_{2}^{2}\right)}{n h_{n}^{d} B_{n, d(1-\ell)}^{2}} \rightarrow 0, \text { as } n \rightarrow \infty .
$$

The last term in (4) is bounded by Billingsley inequality with the help of assumptions $\mathbf{H 2 ( i i i )}$ and H.3(i), as follows.

$$
\begin{aligned}
\Delta_{3} & =2 \sum_{1 \leq i<j \leq r_{n}} \sum_{s=l_{i}} \sum_{t=l_{j}}^{l_{i}+\tau_{n}-1}\left\{\lambda_{1}^{2} \operatorname{Cov}\left(\Psi_{n s}, \Psi_{n t}\right)+\lambda_{2}^{2} \operatorname{Cov}\left(\Psi_{n s}^{\prime}, \Psi_{n t}^{\prime}\right)\right. \\
& \left.+\lambda_{1} \lambda_{2}\left[\operatorname{Cov}\left(\Psi_{n s}, \Psi_{n t}^{\prime}\right)+\operatorname{Cov}\left(\Psi_{n t}, \Psi_{n s}^{\prime}\right)\right]\right\} \\
& \leq \frac{2\left(1+b_{n}\right)^{2}\|K\|_{\infty}^{2} \max \left(\lambda_{1}^{2}, \lambda_{2}^{2}\right) h_{n}^{d(2 \ell-1)}}{n B_{n, d(1-\ell)}^{2}} \sum_{k=1}^{r_{n}-1} \sum_{j=1}^{r_{n}} \sum_{s=l_{j}}^{l_{j}+\tau_{n}-1} \sum_{t=l_{j}}^{l_{j}+\tau_{n}-1}\left(h_{s} h_{t}\right)^{-d \ell} \alpha_{X}\left[k\left(\varsigma_{n}+\tau_{n}\right)\right] \\
& \leq \frac{2 \gamma\left(1+b_{n}\right)^{2}\|K\|_{\infty}^{2} \max \left(\lambda_{1}^{2}, \lambda_{2}^{2}\right) r_{n} \tau_{n}^{2}}{n h_{n}^{d} B_{n, d(1-\ell)}^{2}} \sum_{k=1}^{r_{n}-1} e^{-\rho k \tau_{n}} .
\end{aligned}
$$

Therefore,

$$
\Delta_{3}=O\left\{\frac{b_{n}^{2} r_{n} \tau_{n}^{2} e^{-\rho \tau_{n}}}{n h_{n}^{d} B_{n, d(1-\ell)}^{2}}\left[1-e^{-\rho \tau_{n}\left(r_{n}-1\right)}\right]\right\} \rightarrow 0, \text { as } n \rightarrow \infty,
$$

as long as $b_{n}=(\delta \ln n)^{\frac{1}{\theta}}$ with $\delta>\frac{2}{\lambda}$, and $\theta>1 / \varsigma_{0}$. Now, let us prove that $\mathrm{E} S_{n 3}^{2} \rightarrow 0$ as $n \rightarrow 0$. One has

$$
\mathrm{E} S_{n 3}^{2}=\sum_{j=\bar{N}+1}^{n} \operatorname{Var} \tilde{\Psi}_{n j}+2 \sum_{\bar{N}+1 \leq i<j \leq n} \operatorname{Cov}\left(\tilde{\Psi}_{n i}, \tilde{\Psi}_{n j}\right):=\Theta_{n 1}+\Theta_{n 2} .
$$

The variance term $\Theta_{n 1}$ may be written as

$\Theta_{n 1}=\sum_{j=\bar{N}+1}^{n}\left[\lambda_{1}^{2} \operatorname{Var} \Psi_{n j}+\lambda_{2}^{2} \operatorname{Var} \Psi_{n j}^{\prime}+2 \lambda_{1} \lambda_{2} \operatorname{Cov}\left(\Psi_{n j}, \Psi_{n j}^{\prime}\right)\right]:=\lambda_{1}^{2} \Theta_{n 11}+\lambda_{2}^{2} \Theta_{n 12}+2 \lambda_{1} \lambda_{2} \Theta_{n 13}$.

The first term of the right hand side of the preview decomposition satisfies the relation

$$
n h_{n}^{d} \operatorname{Var} f_{n}^{\ell}(x) \sim \sum_{j=1}^{n} \operatorname{Var}\left(\Psi_{n j}\right)=\sum_{j=1}^{\bar{N}} \operatorname{Var}\left(\Psi_{n j}\right)+\Theta_{n 11}
$$

However, one may write

$$
\sum_{j=1}^{\bar{N}} \operatorname{Var}\left(\Psi_{n j}\right)=\left(\frac{n h_{n}^{d}}{\bar{N} h_{\bar{N}}^{d}}\right) \bar{N} h_{\bar{N}}^{d} \operatorname{Var} f_{\bar{N}}^{\ell}(x) .
$$


Since $\bar{N} \sim n$, the condition $u_{n} \sim v_{n}$ implies $h_{u_{n}} \sim h_{v_{n}}$, which leads to $n h_{n}^{d} \sim \bar{N} h_{\bar{N}}^{d}$, which together with Lemma 1(c) imply that $\sum_{j=1}^{\bar{N}} \operatorname{Var}\left(\Psi_{n j}\right) \rightarrow \sigma_{\ell}^{2}(x)$, as $n \rightarrow \infty$. It follows that $\Theta_{n 11}=o(1)$, because $\sum_{j=1}^{n} \operatorname{Var}\left(\Psi_{n j}\right) \rightarrow \sigma_{\ell}^{2}(x)$, as $n \rightarrow \infty$. Let us mention that if $\ell \geq 1 / 2$, then the condition $u_{n} \sim v_{n}$ implies $h_{u_{n}} \sim h_{v_{n}}$, is not necessary. Indeed, the variance term $\Theta_{n 1}$ can be written as

$$
\Theta_{n 1}=\frac{B_{n, d(1-\ell)}^{-2}}{n} \sum_{i=\bar{N}+1}^{n}\left(\frac{h_{i}}{h_{n}}\right)^{d(1-2 \ell)} h_{i}^{-d} \operatorname{Var}\left\{K\left(\frac{x-X_{i}}{h_{i}}\right)\left(1+m\left(Y_{i}\right) \mathbf{1}_{\left|m\left(Y_{i}\right)\right| \leq b_{n}}\right)\right\} .
$$

Since $h_{n}$ is decreasing and $\ell \geq \frac{1}{2}$, then the Toeplitz lemma, with the help of assumption H.2(ii) and the convergence

$$
h_{i}^{-d} \operatorname{Var} K\left(\frac{x-X_{i}}{h_{i}}\right) \rightarrow f(x) \int_{\mathbb{R}^{d}} K^{2}(x) d x, \text { as } i \rightarrow \infty
$$

imply that

$$
\Theta_{n 1} \leq \frac{\operatorname{Cste}(n-\bar{N})\left(1+b_{n}\right)^{2}}{n B_{n, d(1-\ell)}^{2}}
$$

Because of $n-\bar{N} \leq \varsigma_{n}+\tau_{n}$, it follows that $\Theta_{n 1} \rightarrow 0$ as $n \rightarrow \infty$, provided $b_{n}=(\delta \ln n)^{\frac{1}{\theta}}$ with $\delta>$ $\frac{2}{\lambda}$, and $\theta>1 / \varsigma_{0}$. Also, in the same manner, and by replacing $f_{n}^{\ell}$ by $\tilde{\varphi}_{n}^{\ell}$, we can deduce from Lemma 2(a) that $\Theta_{n 12}=o(1)$. Finally, the last term $\Theta_{n 13}$ is bounded similarly to the first term by using Lemma 2(b). Therefore, from $\Theta_{n 13}=o(1)$, it follows that $\Theta_{n 1} \rightarrow 0$ as $n \rightarrow \infty$. Now, let us study the term $\Theta_{n 2}$ in (5). This can be decomposed as

$$
\Theta_{n 2}=2 \sum_{\bar{N}+1 \leq i<j \leq n}\left[\lambda_{1}^{2} \operatorname{Cov}\left(\Psi_{n i}, \Psi_{n j}\right)+\lambda_{2}^{2} \operatorname{Cov}\left(\Psi_{n i}^{\prime}, \Psi_{n j}^{\prime}\right)+2 \lambda_{1} \lambda_{2} \operatorname{Cov}\left(\Psi_{n i}, \Psi_{n j}^{\prime}\right)\right] .
$$

As in the proof of Lemma 2, one may show that

$$
\sum_{\bar{N}+1 \leq i<j \leq n}\left[\lambda_{1}^{2} \operatorname{Cov}\left(\Psi_{n i}, \Psi_{n j}\right)+\lambda_{2}^{2} \operatorname{Cov}\left(\Psi_{n i}^{\prime}, \Psi_{n j}^{\prime}\right)\right] \rightarrow 0, \text { as } \rightarrow 0
$$

and

$$
\sum_{\bar{N}+\leq i<j \leq n} \operatorname{Cov}\left(\Psi_{n i}, \Psi_{n j}^{\prime}\right) \leq \sum_{1 \leq i<j \leq n} \operatorname{Cov}\left(\Psi_{n i}, \Psi_{n j}^{\prime}\right) \rightarrow 0, \text { as } \rightarrow 0 .
$$


Hence, $\Theta_{n 2} \rightarrow 0$, as $\rightarrow 0$. To complete the proof we must show that the asymptotic distribution of $S_{n 1}$ is normal. To this end let us check the Lindeberg-Feller conditions for $S_{n 1}$. First, we consider a sequence of iid random variables $Z_{n 1}, \ldots, Z_{n r_{n}}$, having the same distribution as $T_{n m}$. Then, $\mathrm{E} Z_{n 1}=0$ and if $\Phi_{T_{n m}}$ is the characteristic function (f.c.) of $T_{n m}$, then $\Phi_{T_{n m}}^{r_{n}}$ is the f.c. of the random variable $\sum_{m=1}^{r_{n}} Z_{n m}$. To establish the asymptotic normality of $S_{n 1}$, it suffices to prove that the variables $\sum_{m=1}^{r_{n}} Z_{n m}$ and $\sum_{m=1}^{r_{n}} T_{n m}$ have the same distribution, and that this latter is Gaussian. By the Volkonskii-Rosanov [18] lemma, one has

$$
\left|\mathrm{E} \prod_{m=1}^{r_{n}} e^{i t T_{n m}}-\prod_{m=1}^{r_{n}} \mathrm{E} e^{i t T_{n m}}\right| \leq 8\left(r_{n}-1\right) \alpha\left(\tau_{n}\right) \leq \rho_{0} r_{n} e^{-\rho_{1} \tau_{n}} \rightarrow 0, \text { as } n \rightarrow \infty .
$$

It follows that $\left|\mathrm{E} \prod_{m=1}^{r_{n}} e^{i t T_{n m}}-\Phi_{T_{n}}^{r_{n}}\right| \rightarrow 0$, as $n \rightarrow \infty$. Then, it suffices to prove that $\Phi_{T_{n m}}^{r_{n}}$ converges to the characteristic function of a Gaussian random variable. To this end, we proceed as follows. Set $Z_{n m}^{\prime}:=\frac{Z_{n m}}{s_{n}}$, where $s_{n}^{2}:=\sum_{m=1}^{r_{n}} \operatorname{Var} Z_{n m}$. One has $s_{n}^{2} \rightarrow \Sigma_{\ell}^{2}(x)$, as $n \rightarrow$ $\infty$. Indeed, $s_{n}^{2}=\sum_{m=1}^{r_{n}} \operatorname{Var} T_{n m} \rightarrow \Sigma_{\ell}^{2}(x)$, as $n \rightarrow \infty$, because on one hand we have from Lemmas 1 and 2 :

$\operatorname{Var} S_{n 1} \sim n h_{n}^{d}\left\{\lambda_{1}^{2} \operatorname{Var} f_{n}^{\ell}(x)+\lambda_{2}^{2} \operatorname{Var} \tilde{\varphi}_{n}^{\ell}(x)+2 \lambda_{1} \lambda_{2} \operatorname{Cov}\left[f_{n}^{\ell}(x), \tilde{\varphi}_{n}^{\ell}(x)\right]\right\} \rightarrow \Sigma_{\ell}^{2}(x)$, as $n \rightarrow \infty$ and one may show, on the other hand, as for $\Delta_{2}$, that $\sum_{1 \leq i<j \leq r_{n}} \operatorname{Cov}\left(T_{n i}, T_{n j}\right) \rightarrow 0$, as $n \rightarrow \infty$. Hence, the variables $Z_{n m}^{\prime}$ are iid, $\mathrm{E} Z_{n 1}^{\prime}=0$ and $\sum_{m=1}^{r_{n}} \operatorname{Var} Z_{n m}^{\prime}=1$. By virtue of the Lindeberg conditions (c.f. Loève [13]), we have to show that for all $\varepsilon>0$,

$$
\sum_{m=1}^{r_{n}} \mathrm{E}\left(Z_{n m}^{\prime 2} \mathbf{1}_{\left\{\left|Z_{n m}^{\prime}\right|>\varepsilon\right\}}\right) \rightarrow 0, \text { as } n \rightarrow \infty .
$$

Noting that $\left|T_{n m}\right| \leq \frac{\varsigma_{n}\|K\|_{\infty}\left(1+b_{n}\right)}{\sqrt{n h_{n}^{d}} B_{n, d(1-\ell)}}$, and applying Markov's inequality, one has

$$
\begin{aligned}
\sum_{m=1}^{r_{n}} \mathrm{E}\left(Z_{n m}^{\prime 2} \mathbf{1}_{\left\{\left|Z_{n m}^{\prime}\right|>\varepsilon\right\}}\right) & =\sum_{m=1}^{r_{n}} \mathrm{E}\left(\frac{T_{n m}^{2}}{s_{n}^{2}} \mathbf{1}_{\left\{\left|T_{n m}\right|>\varepsilon s_{n}\right\}}\right) \leq \frac{\varsigma_{n}^{2}\left(1+b_{n}\right)^{2}\|K\|_{\infty}^{2}}{n h_{n}^{d} B_{n, d(1-\ell)^{2}}^{2}} \sum_{m=1}^{r_{n}} P\left(\left|T_{n m}\right|>\varepsilon s_{n}\right) \\
& \leq\left[\frac{\varsigma_{n}\left(1+b_{n}\right)}{\sqrt{n h_{n}^{d}}} \cdot \frac{\|K\|_{\infty} \varepsilon^{-1}}{s_{n} B_{n, d(1-\ell)}}\right]^{2} \rightarrow 0, \text { as } n \rightarrow \infty,
\end{aligned}
$$

if $b_{n}=(\delta \ln n)^{\frac{1}{\theta}}$ with $\delta>\frac{2}{\lambda}$, and $\theta>1 / \varsigma_{0}$. 


\section{References}

[1] Ahmad, I. and Lin, P.E. (1976). Nonparametric sequential estimation of a multiple regression function, Bull. Math. Statist. 17, 63-75.

[2] Amiri, A. (2009). Sur une famille paramétrique d'estimateurs séquentiels de la densité pour un processus fortement mélangeant, C. R. Acad. Sci. Paris, Ser. I, 347, 309-314.

[3] Amiri, A. (2012). Recursive regression estimators with application to nonparametric prediction. J. Nonparam. Statist., 24, 169-186.

[4] Bosq, D. and Blanke, D. (2007). Inference and prediction in large dimensions, Wiley series in probability and statistics.

[5] Bosq, D. and Cheze-Payaud, N. (1999). Optimal asymptotic quadratic error of nonparametric regression function estimates for a continuous-time process from sampled-data, Statistics 32 (3), 229-24\%.

[6] Devroye, L. (1981). On the almost everywhere convergence of nonparametric regression function estimates, Ann. Statist. 9, 1310-1319.

[7] Devroye, L. and Wagner, T. J. (1980). On the $L^{1}$ convergence of kernel estimators of regression functions with application in discrimination, Z. Wahrschein. Verw. Get. 51, 15-25.

[8] Doob, J. (1953). Stochastic process, Wiley New York.

[9] Greblecki, W. and Pawlak, M. (1987). Necessary and sufficient consistency conditions for a recursive kernel regression estimate, J. Multivariate Anal. 23, 67-76.

[10] Gyorfi, L., Hardle, W., Sarda, P. and Vieu, P. (1989). Nonparametric curve estimation from time Series, Lecture notes in statistics, Springer.

[11] Györfi, L., Khöler, M., Krzyżak, A. and Walk, H. (2002). A distribution-free theory of nonparametric regression, Springer-Verlag New york. 
[12] Krzyżak, A. (1992). Global convergence of the recursive kernel regression estimates with applications in classification and nonlinear system estimation, IEEE Trans. Inform. Theory 38, 1323-1338.

[13] Loève, M. (1963). Probability theory, Princeton, New Jersey Van Nostrand.

[14] Prakasa-Rao, B.L.S. (1983), Nonparametric functional estimation, New-York: Academic Press.

[15] Qin, Y.S. (1995), ÔAsymptotic distribution of a recursive kernel estimator for a nonparametric regression function under dependent samplingÕ, Mathematica Applicata, 8(1), 7-13.

[16] Roussas, G.G. (1990). Nonparametric regression estimation under mixing conditions. Stochastic Process. Appl. 36 (1), 107-116.

[17] Roussas, G.G. and Tran, L.T. (1992). Asymptotic normality of the recursive kernel regression estimate under dependence conditions, Annals of Statist. 20 (1), 98-120.

[18] Volkonskii, V.A. and Rozanov, Yu.A. (1959). Some limit theorems for random functions, Theory Probab. Appl. 4, 178-19\%.

[19] Walk, H. (2001). Strong universal pointwise consistency of recursive regression estimates, Ann. Inst. Statist. Math. 53 (4), 691-707.

[20] Wang, L., and Liang, H.Y. (2004), ÔStrong uniform convergence of the recursive regression estimator under $\varphi$-Mixing ConditionsÕ, Metrika, 59(3), 245-261.

[21] Yoshihara, K. (1994), Weakly dependent stochastic sequences and their applications: curve estimation based on weakly dependent data (Vol. IV), Tokyo: Sanseido. 